\title{
Protective Effect and Mechanism of Shengmai Formula in Ultrafine Particle Matter-Mediated Atherosclerosis and Myocardial Ischemic Injury
}

\section{Ting Yang}

China Academy of Chinese Medical Sciences

\section{Shuiqing Qu}

China Academy of Chinese Medical Sciences

\section{Yuanmin Yang}

China Academy of Chinese Medical Sciences

\section{Yu Zhang}

China Academy of Chinese Medical Sciences

\section{Shuoqiu Deng}

China Academy of Chinese Medical Sciences

\section{Zhongyuan Zheng}

China Academy of Chinese Medical Sciences

Hui Liu

China Academy of Chinese Medical Sciences

\section{Xi Wang}

China Academy of Chinese Medical Sciences

\section{Lina Chen}

China Academy of Chinese Medical Sciences

\section{Xiaoxin Zhu}

China Academy of Chinese Medical Sciences

\section{Yujie Li ( $\nabla$ yjli@icmm.ac.cn )}

China Academy of Chinese Medical Sciences

\section{Research}

Keywords: Shengmai, Atherosclerosis, Myocardial infarction, Ultra fine particulate matter, Oxidative stress, Keap1-Nrf2/HO-1 pathways.

Posted Date: April 6th, 2021

DOI: https://doi.org/10.21203/rs.3.rs-384449/v1 
License: (c) (i) This work is licensed under a Creative Commons Attribution 4.0 International License. Read Full License 


\section{Abstract}

Background: Long-term exposure to ultrafine particulate matter (UFPM) has been shown to enhance the development of atherosclerosis and impair myocardial function, leading to myocardial infarction (MI). The Shengmai (SM) formula is a kind of traditional Chinese medicine for cardiovascular diseases, which has been used in clinic for thousands of years. It is composed of Red ginseng, dwarf lilyturf tuber (Ophiopogon japonicus), and Chinese magnolia vine fruit and has been reported to improve myocardial ischemia and combat oxidative stress (OS) injury. The aim of this study was to explore the protective role of SM and the mechanism by which it acts against UFPM-induced cardiovascular injury. This study is expected to provide a basis for the development of potential adaptive signature of SM in the prevention of atherosclerotic cardiovascular disease (ASCVD) and premature death from global air pollution exposure.

Results: Pretreatment with SM reduced plaque vulnerability, improved cardiac systolic function, decreased the OS response, and attenuated UFPM-induced reactive oxygen species production and mitochondrial damage. In addition, mice pretreated with SM showed a smaller MI area. In vitro data further demonstrated that compared with the UFPM group, pretreatment with SM significantly increased the expression of nuclear factor erythroid 2-related factor2 (Nrf2) and HO-1, and to some extent protected the antioxidant defense system.

Conclusion: These results indicate that SM can inhibit the OS reaction in myocardial infarction mice, avoid cardiovascular injury induced by UFPM in atherosclerosis (AS)+MI mice, and improve cardiac function. Furthermore, the Keap1-Nrf2/HO-1 antioxidant signaling pathways might be involved during these processes. It is suggested that SM has a potential protective effect on the ASCVD population with prolonged exposure to excess particulate matter concentration.

\section{Full Text}

This preprint is available for download as a PDF.

\section{Figures}


A

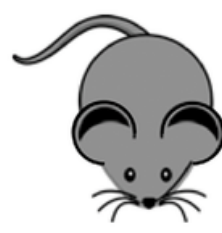

ApoE-/-mice

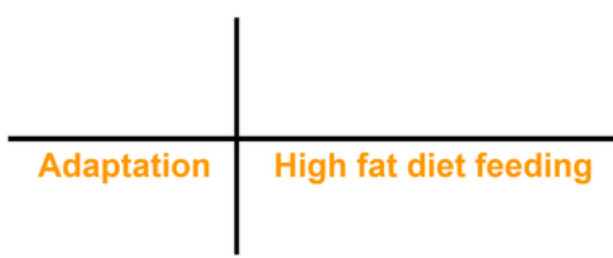

Week 1

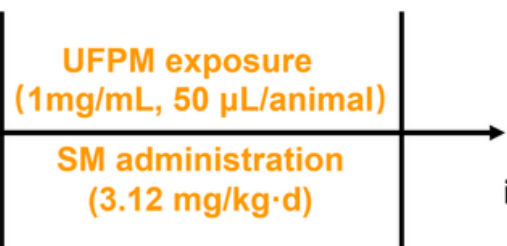

Week 12
LAD ligation and echocardiography, sacrifice for lipid levels assessment, immunohistochemic al analysis, etc.

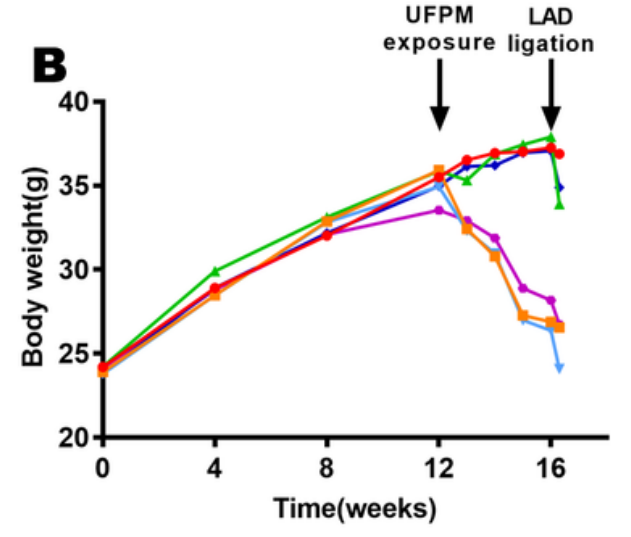

D

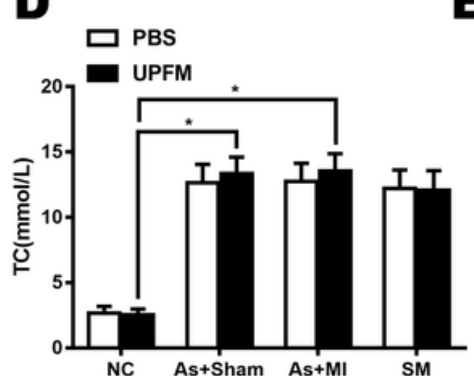

$\rightarrow$ As+Sham(PBS)

-- As+Sham(UFPM)

$=$ As+MI(PBS)

- As+MI(UFPM)

$\rightarrow$ SM(PBS)

$\rightarrow$ SM(UFPM)
E

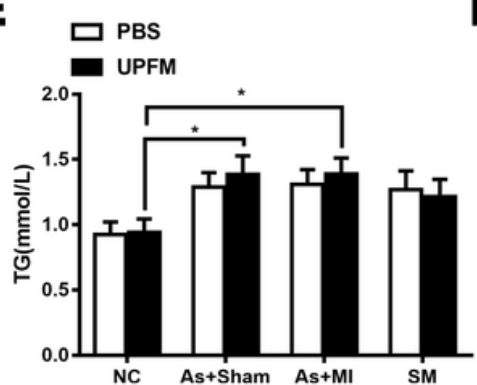

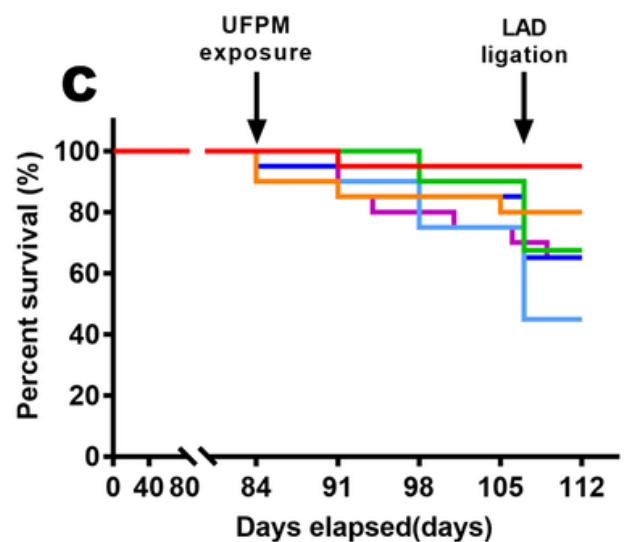

- AS+Sham (PBS)

- AS+Sham (UFPM)

- AS+MI (PBS)

- AS+MI (UFPM)

- SM (PBS)

- SM (UFPM)
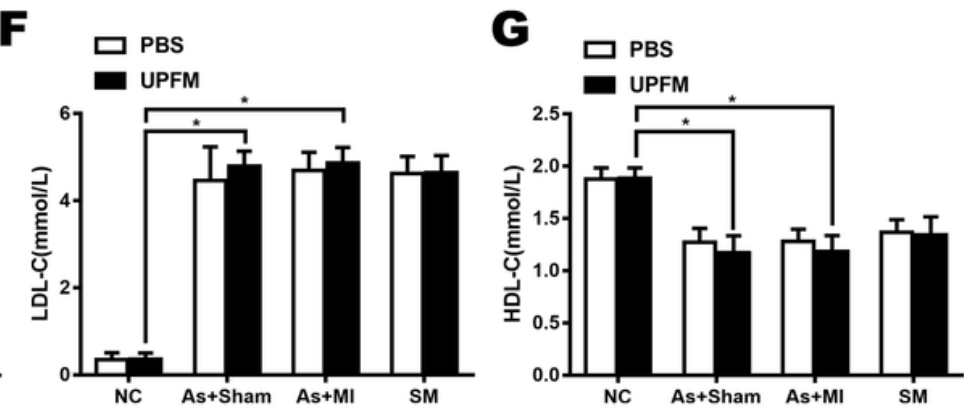

Figure 1

General condition of mice after UFPM and SM intervention. (A) After 12-week of HFD feeding, mice were administered SM (3.12 mg/kg/day) and exposed to particulate matter via inhalation once a week for four consecutive weekdays. At the end of the day, a LAD ligation operation was induced in the coronary artery. (B) Body weight before and after UFPM exposure and LAD ligation was recorded in indicated groups ( $\mathrm{n}=$ $15, * P<0.05, * \star P<0.01)(C)$ Survival curves of mice after UFPM exposure and LAD ligation. $(n=15, * P<$ $\left.0.05,{ }^{\star} \mathrm{P}<0.01\right)(\mathrm{D}-\mathrm{G})$ Effect of UFPM and SM on the serum levels of TC (C), TG (D), LDL (E) and HDL (F) levels in mice. $\left(n=6,{ }^{*}<<0.05,{ }^{\star} \mathrm{P}<0.01\right)$ UFPM: ultrafine particulate matter; $S M$ : Shengmai Formula; HFD: high-fat diet; LAD: left anterior descending; TC: total cholesterol; TG: triglyceride; LDL: low-density lipoprotein-cholesterol; HDL: high-density lipoprotein-cholesterol 

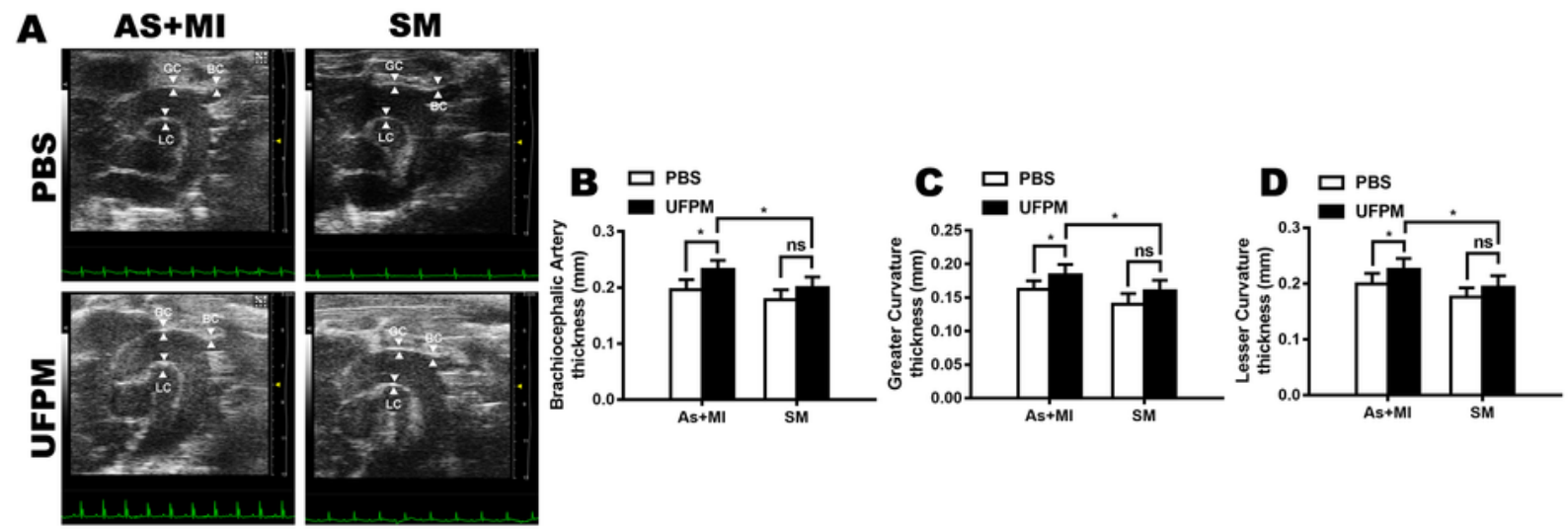

$\mathbf{E}$

AS+MI
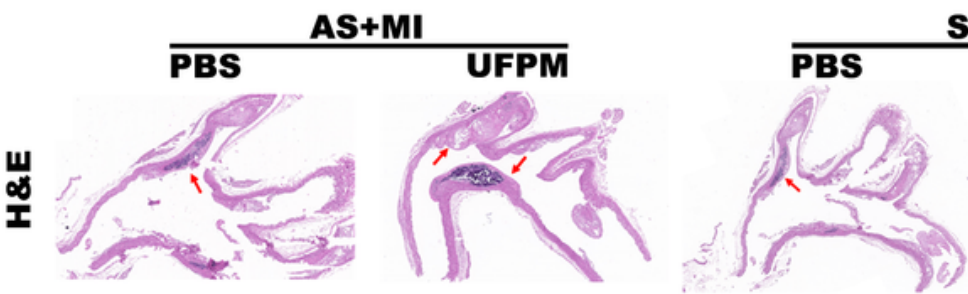

SM
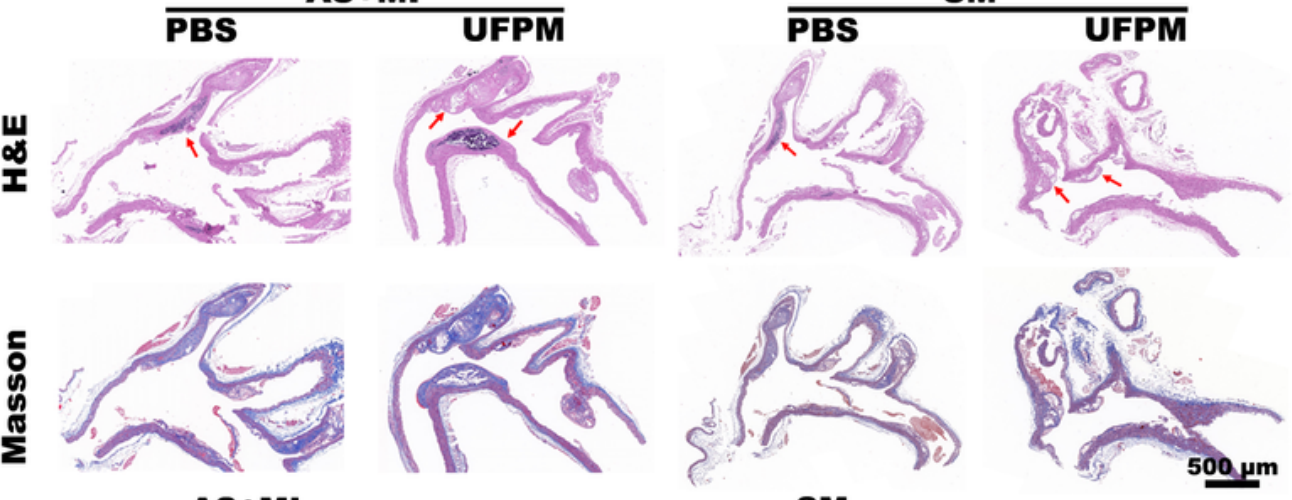

$\mathbf{F}$

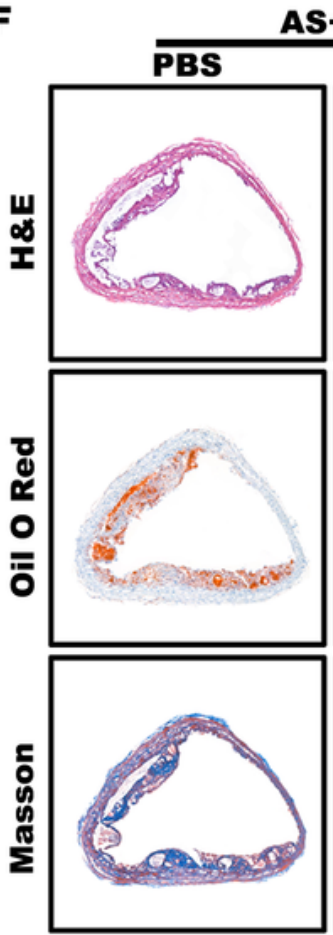

AS+MI
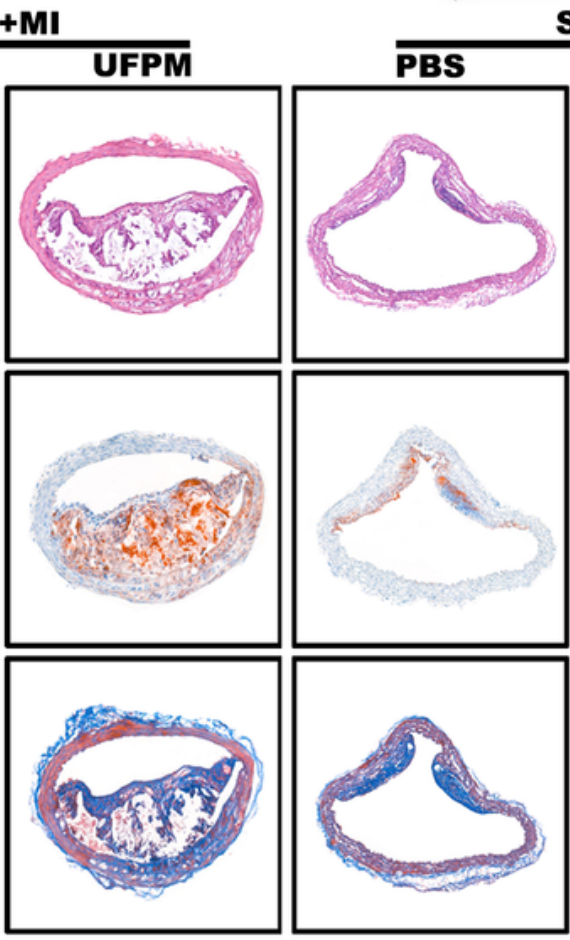

SM
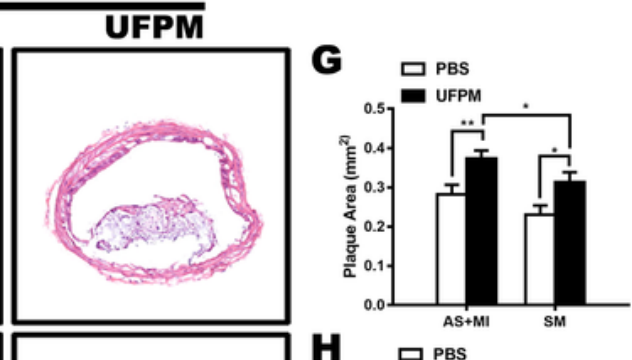

H

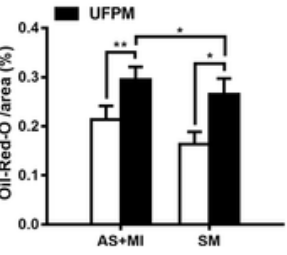

I

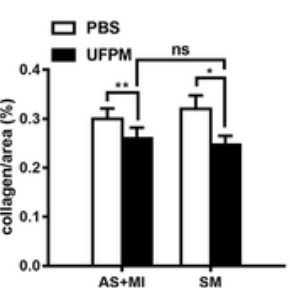

Figure 2

SM reverses fine particle matter -induced AS development (A-D) ultrasound images of the aortic arch in ApoE-/- mice. Wall thickness of the aortic arch was observed at the greater curvature (GC), the lesser curvature (LC) and the origin of the brachiocephalic artery (BC). Values are expressed as mean $\pm S D(n=$ $\left.6,{ }^{*} P<0.05,{ }^{*} \mathrm{P}<0.01\right)$ Thickened wall of the aortic arch sections were observed by ultrasound and indicated with white arrows in representative pictures. $\left(n=6\right.$ per group, $\left.p^{*}<0.05, p^{\star *}<0.01\right)$. 
Representative images of H\&E and Masson staining of longitudinal section of aortic arch; $(\mathrm{n}=6 \mathrm{per}$ group, $\left.\mathrm{p}^{\star}<0.05, \mathrm{p}^{\star \star}<0.01\right)$. (F) Representative images of H\&E, Masson, and Oil Red $\mathrm{O}$ staining of the transverse section of the aortic root; bar $=500 \mu \mathrm{m}$. $\left(n=6\right.$ per group, $\left.\mathrm{p}^{\star}<0.05, \mathrm{p}^{\star \star}<0.01\right)$. (G-I) Statistical analyses of plaque area, lipid content, and collagen area in aortic roots. Values are expressed as mean \pm SD $\left(n=6\right.$ per group, $\left.p^{\star}<0.05, p^{* *}<0.01\right)$. H\&E: hematoxylin and eosin; SM: Shengmai Formula; SD:

standard deviation

A
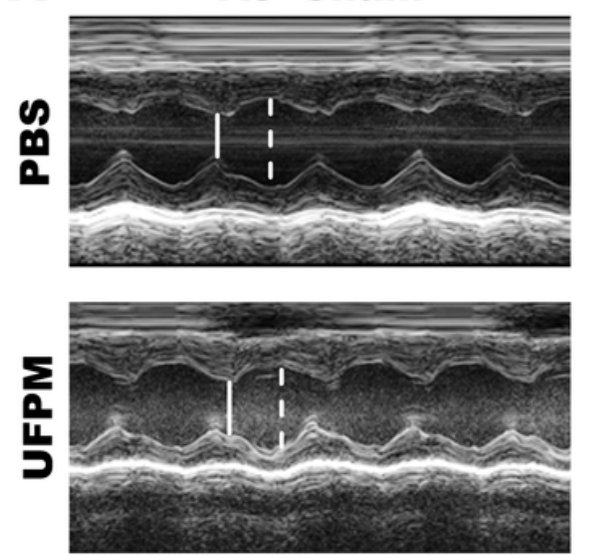

As+MI
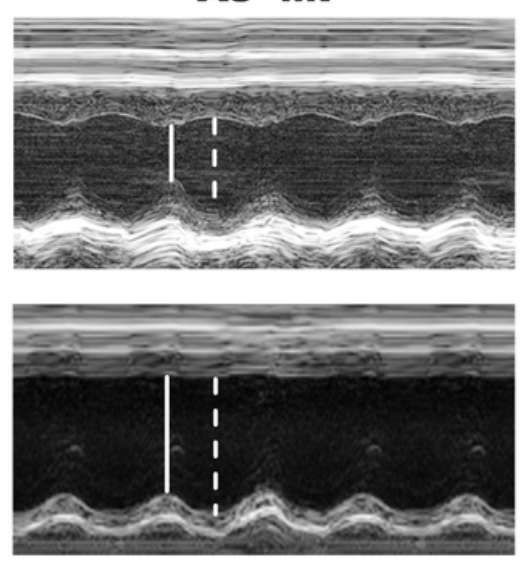

B 마 PBS

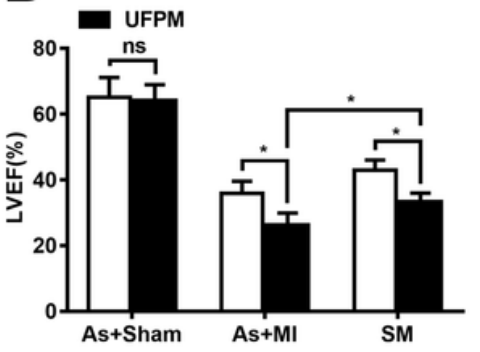

F $\square$ PBS

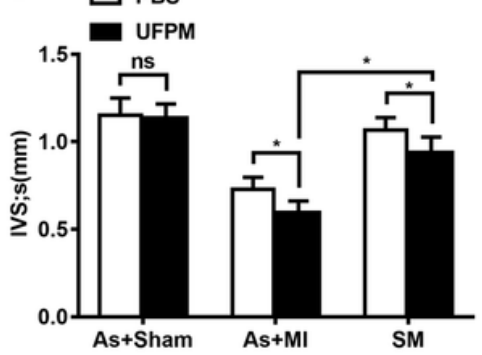

J 口PBS

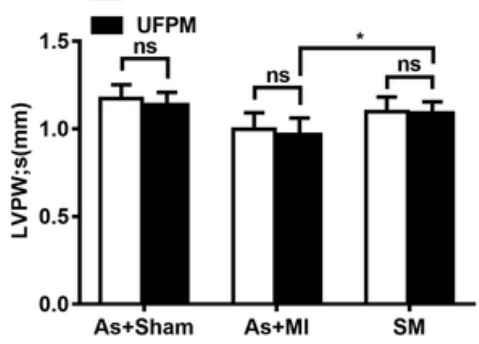

C 모 PBS

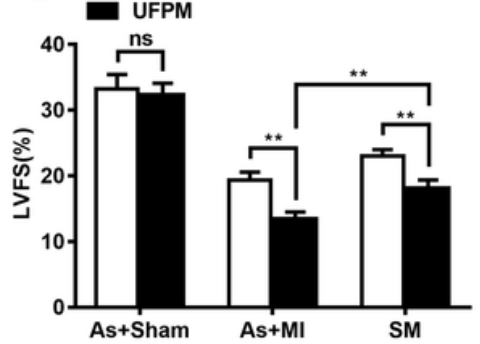

G

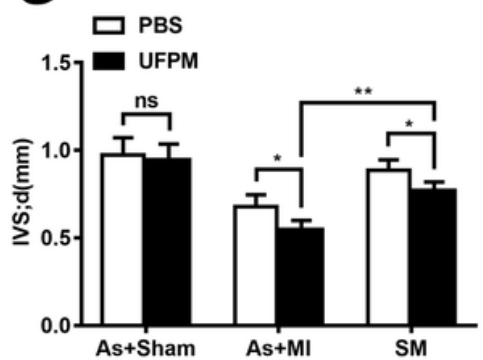

$\mathbf{K}$

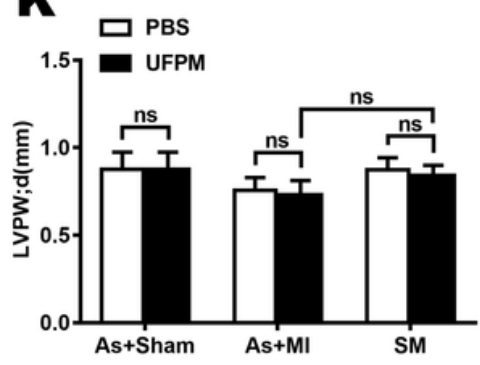

D

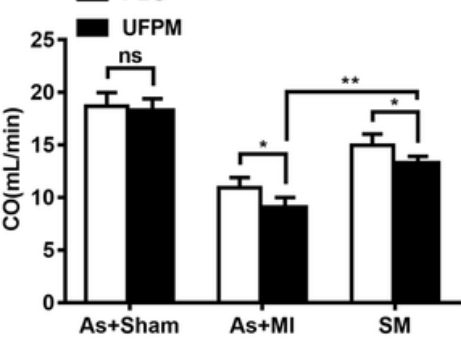

$\mathbf{H}$

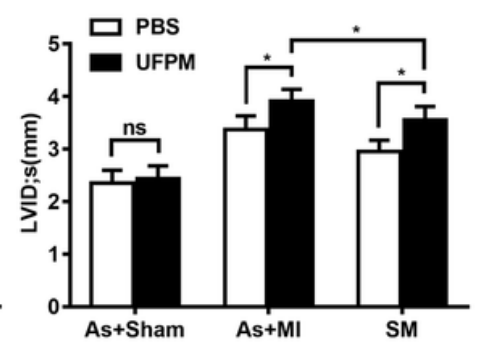

$\mathbf{L}$ 므 $\mathrm{PBS}$

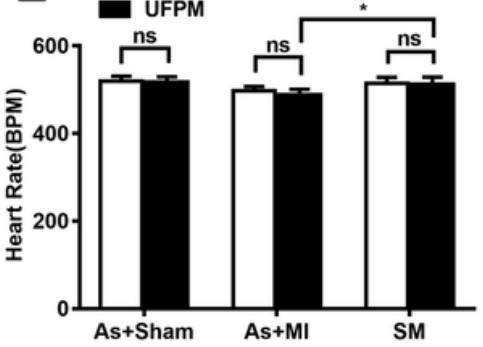

SM
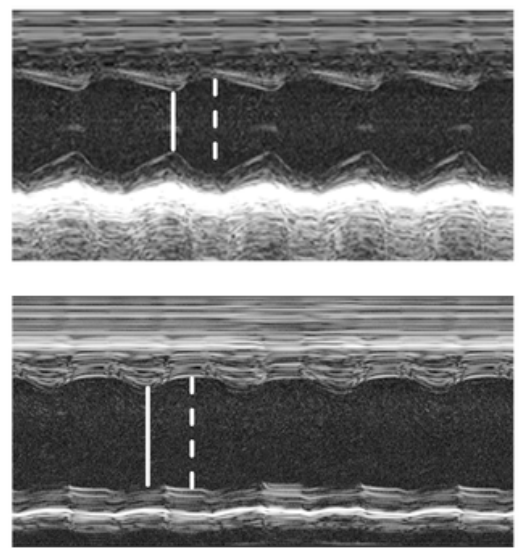

E므 PBS

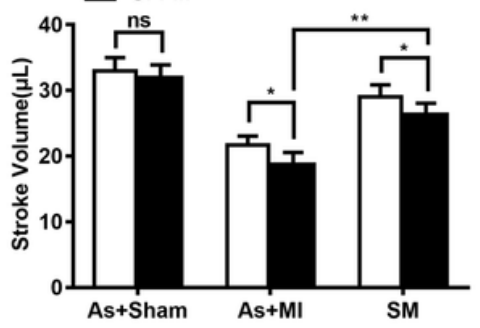

I

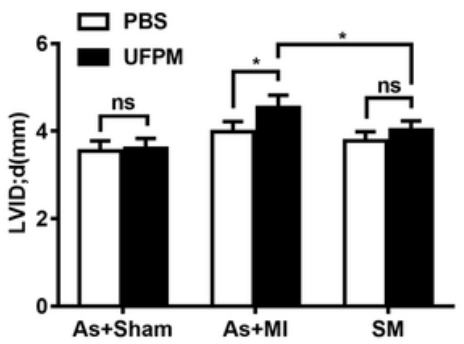

M

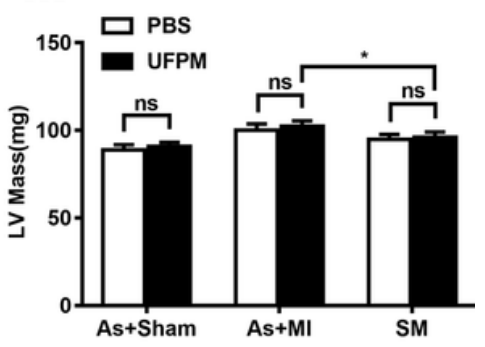

Figure 3

Changes of echocardiographic characterization of cardiac function in myocardial ischemia (MI) mice. After $24 \mathrm{~h}$ of ischemia, the effect of SM (SM, $3.12 \mathrm{mg} / \mathrm{kg} /$ day) on cardiac function was quantitatively 
evaluated. (A) Representative echocardiography images of different groups. Bar graph quantitation of echocardiographic changes in cardiac function in different groups detected in M-mode: (B) LVEF \%, (C) LVFS \%, (D) CO, (E) stroke volume, (F) IVSs, (G) IVSd, (H) LVIDs, (I) LVIDd, (J) LVPWd, (K) LVPWs, (L) Heart Rate, and (M) LV mass. Values were expressed as mean SD $\left(n=6,{ }^{*} P<0.05,{ }^{*} P<0.01\right)$ LV: left ventricular; EF: ejection fraction; FS: LV fractional shortening; CO: cardiac output; IVSd: interventricular septum dimension diastole; IVSs: interventricular septum dimension systole; LVIDd: LV internal dimensions at diastole; LVIDs: LV internal diameter systole; LVPWd: LV posterior wall diastole; LVPWs: LV posterior wall systole.

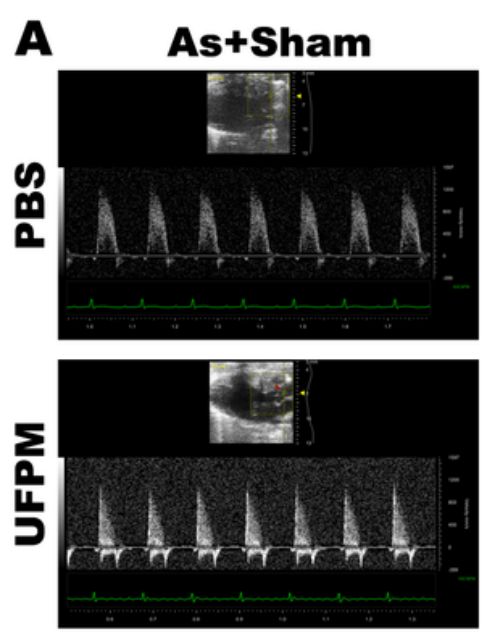

D As+Sham
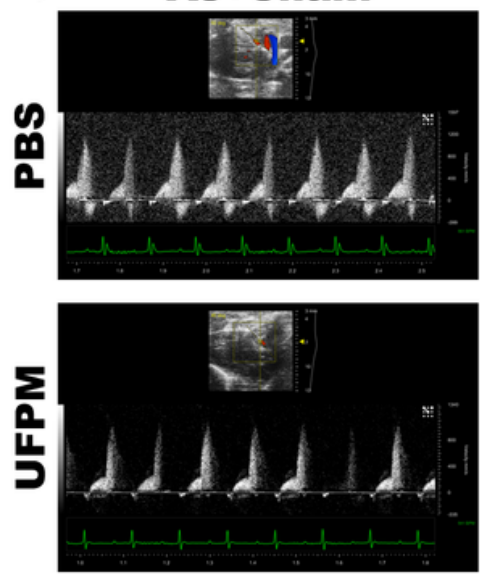

As+MI
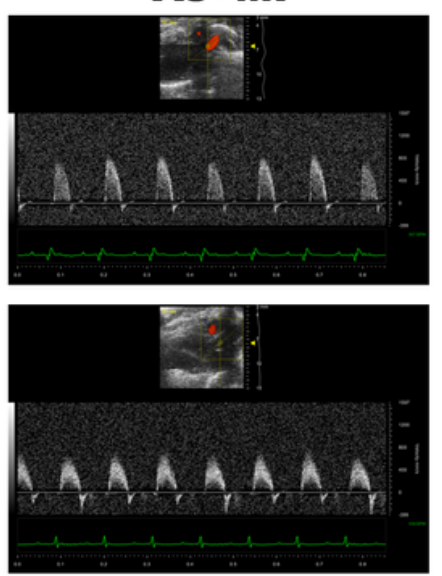

As+MI
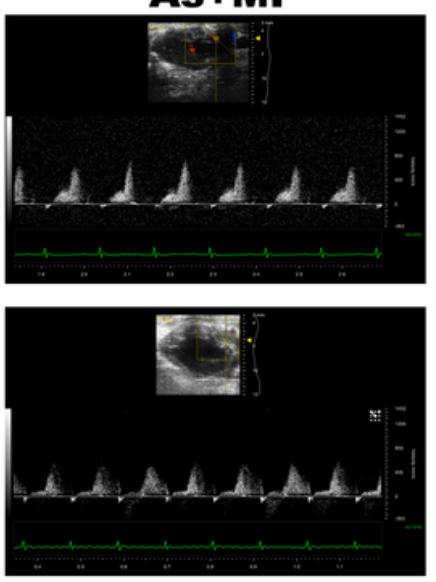
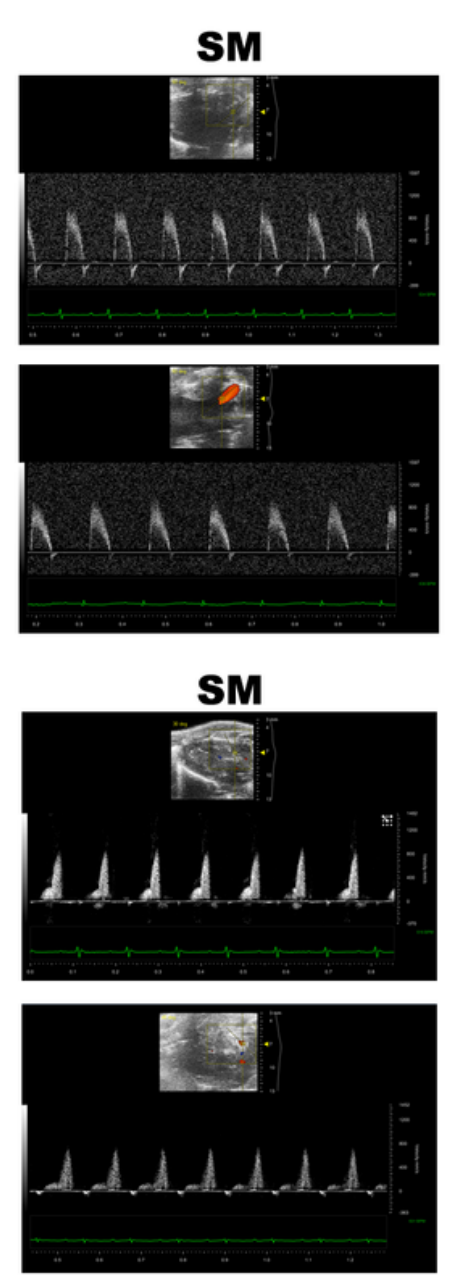
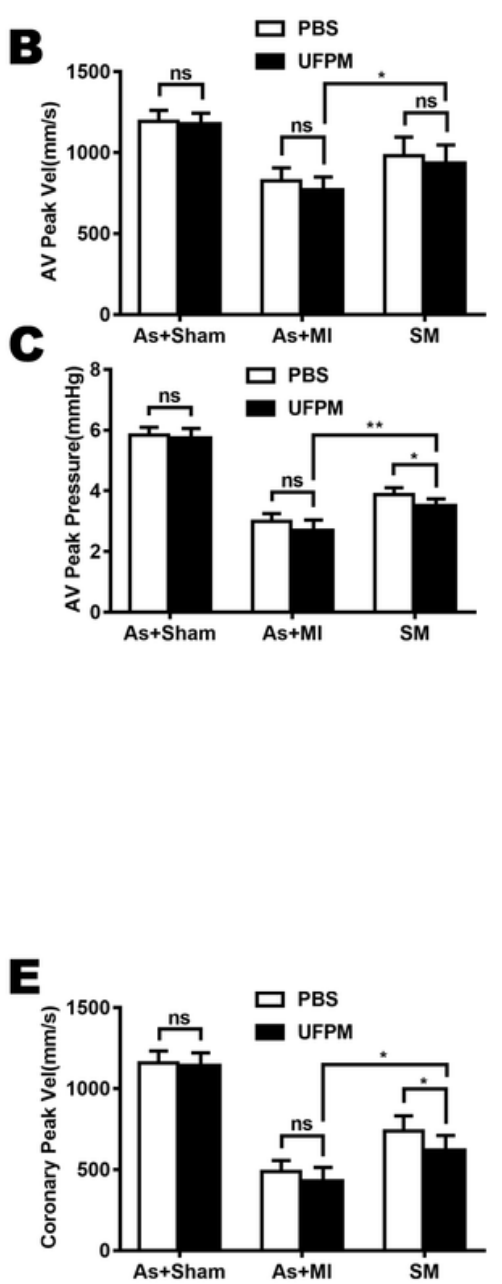

\section{Figure 4}

SM improves the coronary blood flow and left ventricular function after UFPM exposure in MI mice. After $30 \mathrm{~min}$ At $24 \mathrm{~h}$ after ischemia, the effect of SM $(3.12 \mathrm{mg} / \mathrm{kg} /$ day $)$ on cardiac function was quantitatively evaluated. (A) Representative echocardiography images of aortic blood flow were determined with different groups. (B) AV peak velocity and (C) AV peak pressure was detected in color Doppler mode. Values are expressed as mean $\pm S D\left(n=6,{ }^{*} P<0.05,{ }^{*} P<0.01\right)$ (D) Representative echocardiography images of coronary artery flow were determined with different groups. (E) Coronary peak pressure was detected in color Doppler mode. Values are expressed as mean $\pm S D\left(n=6,{ }^{\star} P<0.05, * \star P<0.01\right) A V$ : aortic valve; SM: Shengmai Formula 

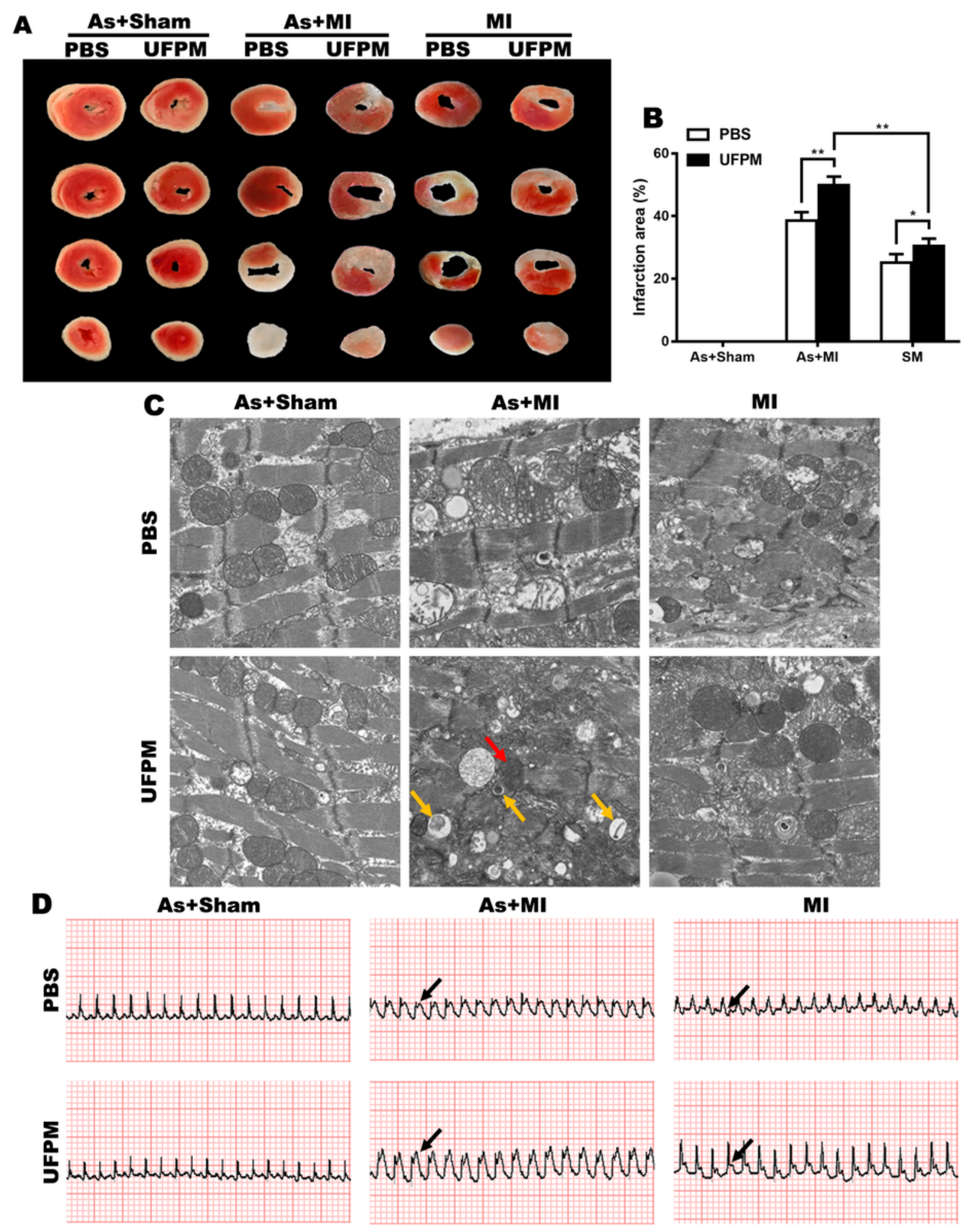

\section{Figure 5}

SM reduces the area of myocardial infarction and preserves the myocardial ultrastructure After $24 \mathrm{~h}$ of ischemia, myocardial tissue was stained by 2, 3, 5-Triphenyl-2H-tetrazolium chloride (TTC). Normal myocardial tissues were represented in red, whereas the white marked areas of infarct. (A) Representative images of TTC staining in different groups, including Sham, MI model, and SM groups. TTC staining was performed at $24 \mathrm{~h}$ after MI. Quantitation of TTC stain of myocardial tissue slices as the percentage of 
infarct volumes of each group $\left(n=6,{ }^{*} P<0.05\right.$, $\left.{ }^{*} P<0.01\right)$ (B) The ultrastructure of mice myocardia was observed under an electron microscopy ( $n=6$, Scale bars $80 \mathrm{um}$ ). (C) Electrocardiogram types before and after UFPM instillation in ApoE-/- mice of each group. $(n=6$, The black arrow represents the abnormal S-T segment) SM: Shengmai Formula; UFPM: ultrafine particulate matter
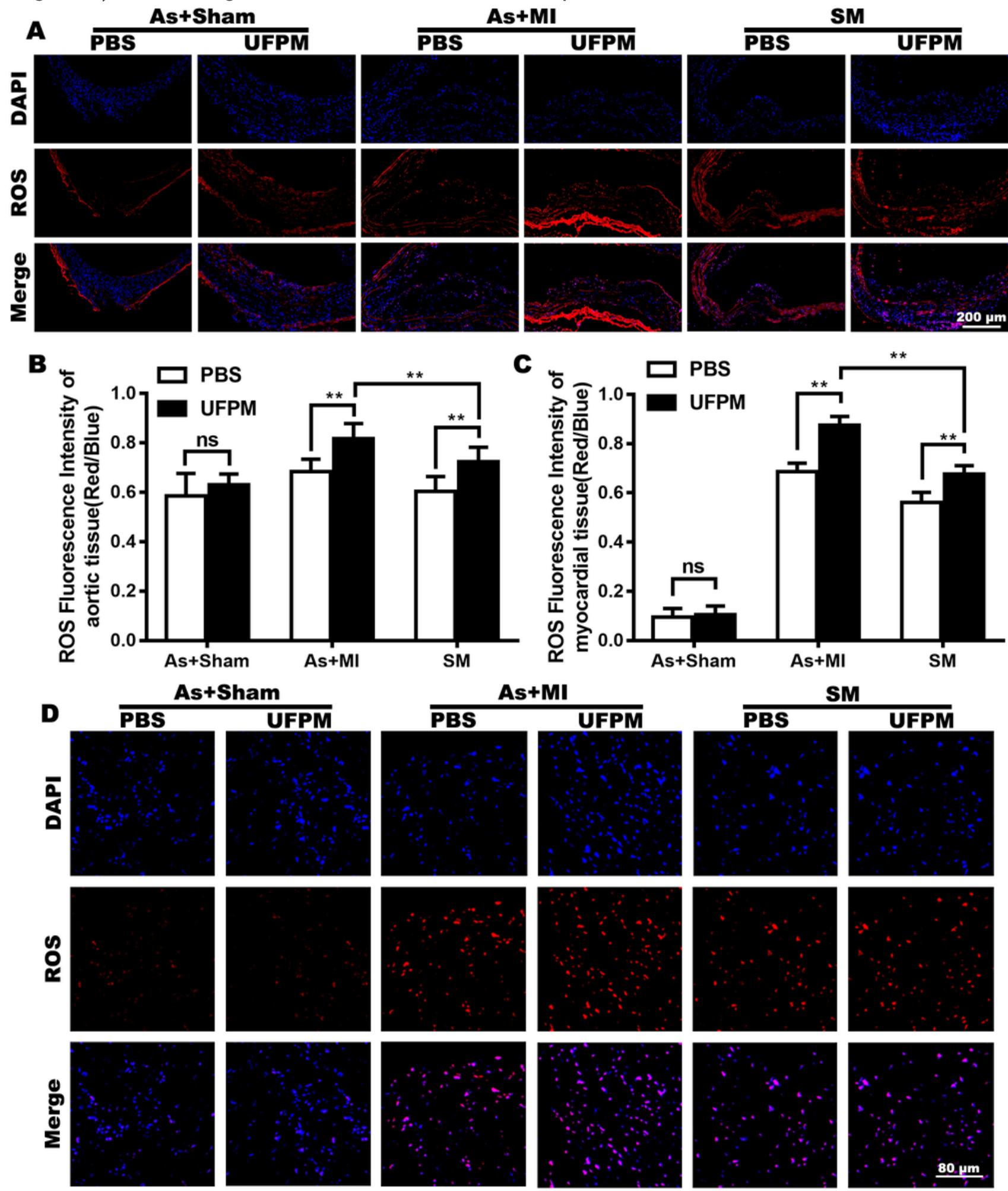

Figure 6 
SM attenuates UFPM-induced OS in MI mice ROS fluorescent probe-DHE. ROS were stained with DHE (red) and nucleus with DAPI (blue). (A and D) UFPM-induced cellular accumulation of reactive oxygen species (ROS) and rescue by SM was detected using ROS fluorescent dye (red color). ROS was induced by UFPM exposure in both the myocardial and vascular tissue of MI mice, and this was ameliorated by treatment with SM. ( $n=6, * P<0.05, * * P<0.01$, Scale bars 200 um and $80 \mathrm{um}$ ). (B and C) ROS accumulation in myocardial and vascular tissue was quantified $(n=6, * P<0.05, * \star P<0.01)$. SM:

Shengmai Formula; UFPM: ultrafine particulate matter; ROS: reactive oxygen species; DAPI: diamidino-2phenylindole: DHE: dihyroethidium
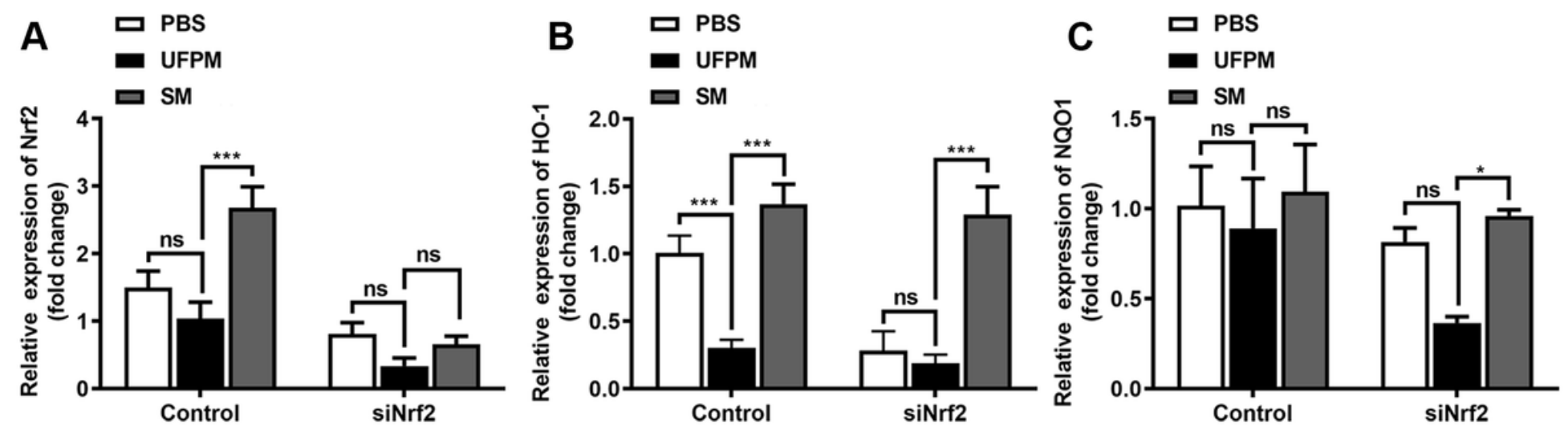

Figure 7

SM plays an antioxidant role in vitro via the Keap1-Nrf2 / HO-1 signal pathway Expression of mRNA levels of Nrf2, HO-1, and NQO1 after UFPM exposure in Control and siNrf2-H9c2 cells. (A)The fold change of Nrf2 in Control and siNrf2-H9c2 cells. ( $\left.n=6,{ }^{*}<<0.05,{ }^{\star} \mathrm{P}<0.01\right)$. (B)The fold change of HO-1 in Control and siNrf2-H9c2 cells. $\left(n=6,{ }^{*} P<0.05,{ }^{*} P<0.01\right)$. (C)The fold change of NQO1 in Control and siNrf2-H9c2 cells. $\left(n=6,{ }^{*}<<0.05,{ }^{\star} P<<0.01\right)$. SM: Shengmai Formula; UFPM: ultrafine particulate matter

\section{Supplementary Files}

This is a list of supplementary files associated with this preprint. Click to download.

- SupplementaryTableS1.docx 